\title{
ReaR

\section{Manejo anestésico para craniectomía descompresiva por malformación de Chiari tipo I en paciente con sospecha de síndrome de activación mastocitaria}

Ortiz Parra F (1), González De La Aleja M (2) Olea Vielba C (2)

(1) FEA Servicio de Anestesiología, Reanimación y Terapia del Dolor Complejo Hospitalario de Toledo, España.

(2) FEA Servicio de Anestesiología, Reanimación y Terapia del Dolor, Hospital Universitario 12 de Octubre, Madrid, España.

\section{Resumen}

La mastocitosis es una patología infrecuente a la que, sin embargo, todo anestesiólogo debe saber hacer frente por las repercusiones clínicas que pueden derivarse de un manejo perioperatorio inadecuado.

Sus manifestaciones clínicas se deben a la liberación de gránulos de histamina, prostaglandinas y otras sustancias vasoactivas contenidas en los mastocitos, desencadenada por multitud de factores y fármacos, muchos de los cuales son habitualmente empleados en el acto anestésico.

Presentamos el caso de una paciente con sospecha de síndrome de activación mastocitaria y múltiples alergias documentadas, sometida a craniectomía descompresiva por una malformación de Chiari tipo 1 con siringomielia.

\section{Introducción}

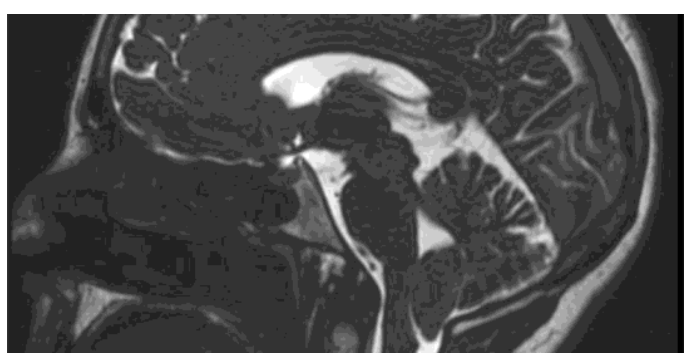

La mastocitosis es una patología infrecuente a la que, sin embargo, todo anestesiólogo debe saber hacer frente por las repercusiones clínicas que pueden derivarse de un manejo perioperatorio inadecuado.

Sus manifestaciones clínicas se deben a la liberación de gránulos de histamina, prostaglandinas $y$ otras sustancias vasoactivas contenidas en los mastocitos, desencadenada por multitud de factores y fármacos, muchos de los cuales son habitualmente empleados en el acto anestésico.

Presentamos el caso de una paciente con sospecha de síndrome de activación mastocitaria y múltiples alergias documentadas, sometida a craniectomía descompresiva por una malformación de Chiari tipo 1 con siringomielia.

Aunque la incidencia exacta de la mastocitosis se desconoce, es una patología infrecuente a la que todo anestesiólogo debe saber hacer frente, por las repercusiones clínicas que pueden derivarse de un manejo perioperatorio inadecuado.

Sus manifestaciones clínicas se deben a la liberación de gránulos de histamina, prostaglandinas $y$ otras sustancias vasoactivas contenidas en los mastocitos, desencadenada por multitud de factores (traumatismos, irritación 
mecánica,

estrés-emociones, irritabilidad, dolor, frío y calor) y por numerosos fármacos, muchos de los cuales son habitualmente empleados en el acto anestésico.

Presentamos el caso de una paciente con sospecha de síndrome de activación mastocitaria y múltiples alergias documentadas, sometida a cirugía programada por una malformación de Chiari tipo 1 con siringomielia.

\section{Caso clínico}

Se trata de una paciente de 42 años con los siguientes antecedentes: preeclampsia en el primer embarazo, y segundo embarazo de curso normal, finalizado con cesárea bajo anestesia general, sin incidencias, por no progresión de parto; fibromialgia con respuesta parcial al tratamiento sintomático, trastorno de ansiedad $\mathrm{y}$ múltiples alergias conocidas (de contacto, alimentarias $\mathrm{y}$ farmacológicas).

Fue evaluada en la consulta preanestésica y por el Servicio de Alergología por sospecha de síndrome de activación mastocitaria.

Analíticamente, presentaba triptasa basal elevada, sin confirmación diagnóstica del síndrome, pero de forma profiláctica se recomendó tratar como tal. Por este motivo, de cara al procedimiento quirúrgico, y habiéndose documentado además alergia a AINEs (excepto ibuprofeno oral), opiáceos, metamizol y butilescopolamina, se realizó prueba de tolerancia a fentanilo, pudiendo usarse con seguridad.

No presentaba predictores de vía aérea difícil.

Teniendo en cuenta todo lo anterior, se elaboró un plan de manejo perioperatorio que llevamos a cabo según describimos a continuación.

Se ingresó a la paciente la noche anterior a la cirugía para premedicación por vía intravenosa y oral según las recomendaciones del Instituto de Estudios de Mastocitosis de Castilla la Mancha (CLMast) y de la Red Española de Mastocitosis (REMA) ${ }^{1}$ : ranitidina $100 \mathrm{mg}$, dexclorfeniramina $5 \mathrm{mg}$, metilprednisolona $1 \mathrm{mg} / \mathrm{kg}$, montelukast $10 \mathrm{mg} \mathrm{VO}, \mathrm{y}$ bromazepam $1.5 \mathrm{mg} \mathrm{VO}$, $\mathrm{y}$ profilaxis antibiótica en planta (cefazolina $2 \mathrm{~g}$ ).

A su llegada al antequirófano, se complementó la premedicación con ansiolisis (midazolam $2 \mathrm{mg}$ ), y se tranquilizó a la paciente.

En quirófano se utilizó monitorización convencional, además de temperatura central por sonda esofágica, tensión arterial invasiva y presión venosa central. Se realizó inducción con atropina $0.6 \mathrm{mg}$, fentanilo $150 \mathrm{mcg}$, etomidato $20 \mathrm{mg}$ y rocuronio $80 \mathrm{mg}$, e intubación sin dificultad.

Constatada su estabilidad hemodinámica y conectada a ventilación mecánica, se canalizaron los accesos vasculares y la arteria. A continuación, se pronó a la paciente en bloque para iniciar la intervención y se conectó al infusor de líquidos calientes y a la manta térmica para conservar una temperatura en torno a $36^{\circ} \mathrm{C}$.

El mantenimiento anestésico se hizo con sevoflurano 3\% para mantener una CAM 0.8-1 y fentanilo en bolos, permaneciendo en todo momento hemodinámica $\mathrm{y}$ respiratoriamente estable, con temperatura $35.6-36^{\circ} \mathrm{C}$.La determinación de niveles de triptasa en sangre mostró los siguientes resultados: basal $12 \mathrm{mcg} / \mathrm{L}$, intraoperatoria 8.05 $\mathrm{mcg} / \mathrm{L}$, y postoperatoria $9 \mathrm{mcg} / \mathrm{L}$. 
Se completó la analgesia con paracetamol, y se administró profilaxis de NVPO con ondansetrón $4 \mathrm{mg}$. Finalmente, se revirtió el bloqueo neuromuscular inicial con sugammadex $150 \mathrm{mg}$, procediendo a una educción $\mathrm{y}$ extubación suaves. La intervención (craniectomía y descompresión de fosa posterior) transcurrió sin incidencias.

\section{Discusión}

La mastocitosis consiste en una alteración de las células madre hematopoyéticas, que lleva a la proliferación y acumulación de mastocitos en uno o más órganos. Se distinguen varios tipos: la más habitual es la cutánea, más frecuente en la edad pediátrica. Un $10 \%$ de estos pacientes pueden tener también clínica sistémica. Los síntomas a nivel sistémico son más frecuentes en adultos, y la presentación clínica es muy variable. Los órganos más frecuentemente afectados son la piel y la médula ósea; más raramente, el tracto gastrointestinal, hígado, bazo y ganglios linfáticos. El diagnóstico de confirmación es histológico.

Los mastocitos contienen gránulos preformados de mediadores químicos como histamina, heparina, triptasa, y también de mediadores sintetizados durante la activación mastocitaria (PGD2, LTC4). Varios factores pueden llevar a su desgranulación y liberación: traumatismos, irritación mecánica, estrés-emociones, dolor, frío y calor; y muchos de los fármacos habitualmente empleados en el acto anestésico.

Según el estudio más completo sobre mastocitosis y anestesia general $^{2}$, el riesgo de presentar síntomas relacionados con la liberación de mediadores fue considerablemente superior con la anestesia general $(6 \%$ de los procedimientos se complicaron con una reacción), y sucedió anafilaxia en 2 de cada 4 pacientes que no habían recibido premedicación; tasas mayores que la incidencia de reacciones de hipersensiblidad relacionadas con la anestesia en la población general. Otros factores de riesgo para anafilaxia fueron cirugía mayor e historia de anafilaxia en general, sin importar cuál fuera su desencandenante.

Por tanto, la anestesia general en pacientes con mastocitosis supone un procedimiento de alto riesgo, que requiere de una planificación cuidadosa y una anticipación de las posibles complicaciones que puedan presentarse.

Aunque el riesgo no se puede cuantificar, la incidencia de anafilaxia en pacientes con mastocitosis es más baja en niños (con formas cutáneas severas) que en adultos (con formas sistémicas indolentes). Los pacientes que nunca han sufrido una anafilaxia o que han tolerado previamente una anestesia general probablemente tengan un bajo riesgo de reacciones perioperatorias. No obstante, sería recomendable hacer pruebas de alergia, sobre todo en pacientes que ya han tenido reacciones durante la anestesia ${ }^{3}$.

Revisaremos las consideraciones más importantes a tener en cuenta.

La mayoría de centros recomiendan premedicación con terapia antimediadores. No hay evidencia del beneficio de ningún régimen específico, pero incluye corticoides, anti-H1, anti$\mathrm{H} 2$ y antileucotrienos. No asocia efectos adversos graves, por lo que el balance riesgo-beneficio es evidentemente favorable a su administración. Es recomendable completar la premedicación con una adecuada ansiolisis con benzodiacepinas.

Consiste en: (tabla 1) 


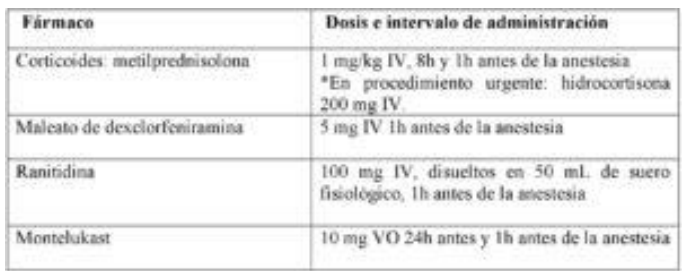

Tabla 1. Premedicación preoperatoria en pacientes con mastocitosis. (adaptado de 1,7)

Tan importante como realizar una correcta selección de los fármacos es minimizar otros factores no farmacológicos que pueden dar lugar a la liberación de histamina: las condiciones ambientales del quirófano temperatura, ruido, características de la comunicación entre los miembros del equipo-, evitar fricción en la piel, etc. Además, es fundamental que exista una comunicación fluida con el paciente, haciéndolo conocedor de su riesgo, pero tranquilizándole y trabajando con él aspectos psicológicos para minimizar su ansiedad.

Centrándonos en el manejo farmacológico, se escogerán siempre aquellos agentes que menor riesgo de desgranulación mastocitaria tengan. No obstante, hay que tener en cuenta que las listas de fármacos a evitar en la mastocitosis se basan en asunciones teóricas y case reports aislados, y que siempre puede desencadenarse una reacción alérgica de novo a cualquier medicación.

Como inductor intravenoso, se recomienda etomidato $0,3 \mathrm{mg} / \mathrm{kg}$, propofol 2-4 mg/kg, o ketamina 1-4.5 $\mathrm{mg} / \mathrm{kg}^{4,5,6}$.

Como relajante neuromuscular, se recomienda vecuronio. Rocuronio también puede emplearse con seguridad. Debemos evitar succinilcolina, atracurio y mivacurio ${ }^{4,6}$.

Para el mantenimiento anestésico, los inhalatorios sevoflurano y desflurano pueden utilizarse ${ }^{5,7}$. Durante este periodo es fundamental conseguir $\mathrm{y}$ asegurar una buena analgesia y profundidad hipnótica, pues tanto el dolor como la superficialización del nivel anestésico pueden desencadenar desgranulación mastocitaria.

Respecto a la analgesia, hay que tener en cuenta que los opioides difieren en su capacidad histaminoliberadora, siendo menor para el fentanilo y sus derivados, y mayor para la morfina. Así pues, se emplearán preferentemente fentanilo y remifentanilo, recomendándose haber constatado previamente que su empleo es seguro. Para analgesia de rescate, paracetamol es una opción válida. El cloruro mórfico y otros opiáceos, como tramadol, y los AINE, están contraindicados.

Con un espectro farmacológico analgésico tan limitado, el control del dolor puede suponer un desafío, por lo que conviene tener presente la posibilidad de realizar bloqueos regionales con anestésicos locales tipo amida.

Ante problemas concomitantes que puedan suceder durante el intraoperatorio, como alteraciones cardiovasculares, debemos saber que también desencadenan la liberación de histamina, y, por tanto, estarían contraindicados, los alfa- bloqueantes y los beta- bloqueantes.

En nuestra paciente, y pese a que los datos sobre su empleo son aún contradictorios, decidimos revertir el bloqueo neuromuscular con sugammadex, sin que aparecieran síntomas derivados de la liberación de histamina. Esta observación tiene gran relevancia clínica, dada la gran utilidad de este fármaco (tabla 2). 


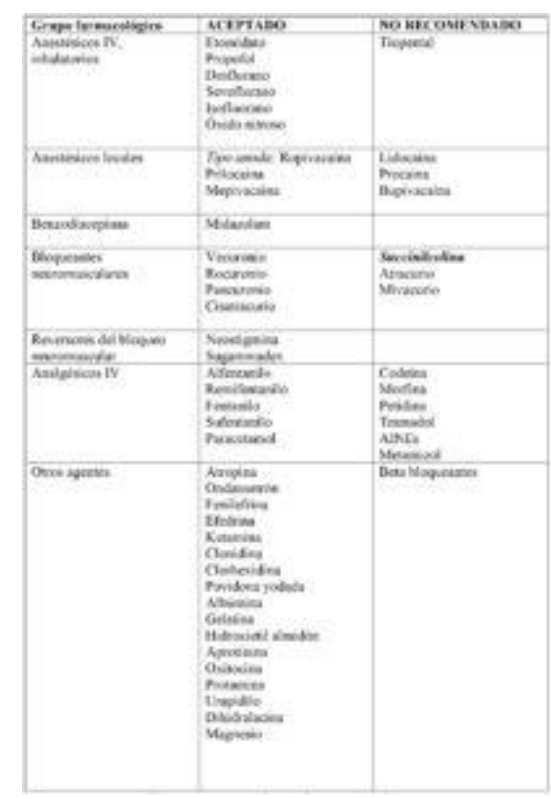

Tabla 2. Empleo de fármacos de uso más común en anestesia en pacientes con mastocitosis (adaptado de 3, 5, 7, 8).

Por último, cabe reseñar que los pacientes con mastocitosis, tanto cutánea como sistémica, pueden tener concomitantemente alergias mediadas por $\operatorname{IgE}$ (rinitis alérgica, alergias alimentarias y medicamentosas, a picadura de insectos). La prevalencia parece ser similar a la de la población general, pero estos pacientes son más susceptibles a la anafilaxia durante las reacciones alérgicas, especialmente en respuesta a las picaduras por himenópteros. Además, se sabe que hay mayor riesgo de anafilaxia en pacientes con triptasa basal elevada. Un estudio prospectivo encontró triptasa basal elevada en el $12 \%$ de los pacientes con historia de reacciones sistémicas a picadura de insectos ${ }^{7}$.

Por ello, y aunque aún se desconoce el valor predictivo de la determinación de triptasa basal en el estudio perioperatorio, sería recomendable extraer una muestra preoperatoria, una intraoperatoria, y una postoperatoria.

\section{Conclusiones}

El riesgo de anafilaxia en pacientes con mastocitosis es generalmente más bajo de lo que la mayoría de los médicos anticipan, pero, no obstante, es mayor que en la población general, y la reacción puede ser más severa. El papel de los fármacos como precipitantes de la anafilaxia está probablemente sobreestimado, y los factores físicos son al menos tan importantes como aquéllos en cuanto a su capacidad para inducir la liberación de mediadores.

Por tanto, aunque los síndromes de activación mastocitaria no son muy frecuentes, la gran trascendencia clínica que puede tener una desgranulación mastocitaria sistémica hace que todo anestesiólogo deba ser conocedor de esta patología y de su correcto manejo.

\section{Bibliografía}

1. Escribano L, Álvarez Twose I, Sánchez Muñoz L, Matito A, Morgado JM. Factores desencadenantes de la liberación de mediadores mastocitarios. Protocolos específicos para situaciones de riesgo. Instituto de Estudios de Mastocitosis de Castilla la Mancha (CLMast), Red Española de Mastocitosis (REMA). Hospital Virgen del Valle, Complejo Hospitalario de Toledo, Servicio de Salud de Castilla la Mancha (SESCAM) ( $\underline{\mathrm{PDF}})$

Disponible

en www.mastocitosis.org/protocolos-detratamiento.html [consultada 29/03/2018]

2. Matito A, Morgado JM, Sánchez-López P, Álvarez-Twose I, Sánchez-Muñoz L, Orfao A, et al. Management of Anesthesia in Adult and Pediatric Mastocytosis: A Study of the Spanish Network on Mastocytosis (REMA) Based on 726 Anesthetic Procedures. Int Arch Allergy Immunol 2015; 167:47-56 (ubMed) (TML)

3. Bonadonna P, Pagani M, Aberer W, Bilò MB, Brockow K, Oude Elberink H, et al. Drug hypersensitivity in clonal mast cell disorders: ENDA/EAACI position paper. Allergy 2015; 70: 755-763. (ubMed) ( 
4. Unterbuchner C, Hierl M, Seyfried T, Metterlein T. Anaesthesia and orphan disease: Rapid sequence induction in systemic mastocytosis. Eur J Anaesthesiol 2017; 34:176183 (

5. Ulbrich F, Engelstädter H, Wittau N, Steinmann D. Anaesthetic management of emergency caesarean section in a parturient with systemic mastocytosis. International Journal of Obstetric Anesthesia (2013) 22, 243254.

6. Calvo N, Telletxea S, Intxaurraga K, Arízaga A. Mastocitosis sistémica: manejo perioperatorio. A propósito de dos casos. Rev Esp Anestesiol Reanim. 2010; 57 (3): 192-194. (HTML)

7. Maud A.W. Hermans; Nicolette J.T. Arends; Roy Gerth van Wijk. Management around invasive procedures in mastocytosis. An update.
Ann Allergy Asthma Immunol 119 (2017) 304309. (HTML)

8. Dewachter P, Castells M.C, Hepner D. L, Mouton-Fraivre C. Perioperative Management of Patients with Mastocytosis. Anesthesiology 2014; 120:753-9. (HTML)

Correspondencia al autor

Fátima Ortiz Parra

fatimaortizparra@gmail.com

FEA Servicio de Anestesiología, Reanimación y Terapia del Dolor.

Complejo Hospitalario de Toledo.

Aceptado para el blog en julio de 2019. 